\title{
Investigating the Emission Characteristics of Single Crystal YAG When Activated by High Power Laser Beams
}

\author{
A. Salimian, ${ }^{\mathrm{Z}}$ J. Silver, ${ }^{*}$ G. R Fern, ${ }^{*}$ H. Upadhyaya, A. Metcalfe, T. G. Ireland, P. Harris, \\ and R. Haghpanahan
}

Centre for Phosphors and Display Materials, Wolfson Centre for Material Processing, Institute for Materials and Manufacturing, Brunel University London, Uxbridge UB8 3PH, United Kingdom

\begin{abstract}
Limitations associated with light emitting diodes (LEDs) operating under high current densities due to the efficiency droop has created a need to look for alternative light sources; here we report investigations on the potential of laser diodes (LDs) for high brightness lighting solutions. High power laser diodes require phosphor targets with certain performance criteria such as high thermal conductivity, efficiency and structural geometry. Here we examine the possibility of using single crystal YAG:Ce phosphor materials as potential targets for generation of light via laser diodes. We report on the emission properties of the crystals with different sizes and examine the effect of laser beam incident angle incident on crystal target emission.

(C) The Author(s) 2016. Published by ECS. This is an open access article distributed under the terms of the Creative Commons Attribution 4.0 License (CC BY, http://creativecommons.org/licenses/by/4.0/), which permits unrestricted reuse of the work in any medium, provided the original work is properly cited. [DOI: 10.1149/2.0271610jss] All rights reserved.

(cc) BY
\end{abstract}

Manuscript submitted August 2, 2016; revised manuscript received September 19, 2016. Published September 29, 2016.

Light emitting diodes (LEDs) are the current lighting technology solution gaining a wide adoption in a variety of commercial applications. They are now the dominant new lighting in office, public and domestic application, in addition they have almost completely covered the automotive head lamp market and now they are finding their way into more sophisticated instruments such as solar simulators. The best commercial LED's efficiencies are now above $60 \%$ and they are the most energy saving solution available. However there is a fundamental issue undermining their performance when they are considered for high brightness lighting applications. To generate high emission intensities, LEDs need to be driven at high current densities at which point their efficiency suffers from a phenomenon described as the efficiency droop. The efficiency droop's origin is rooted in a variety of complex processes including; Auger recombination, overflow of carriers from rich potential minima and carrier leakage out of active region. ${ }^{1-8}$ So far the highest luminous efficacies reported on LEDs are based on driving them at low current densities on the order of $>11 \mathrm{Acm}^{-2}{ }^{9,10}$ In contrast to LEDs, Laser Diodes (LDs) can operate at very high current densities without suffering from efficiency droop. ${ }^{11,12}$ However currently the LD's overall efficiency is well below that of LEDs and their application in lighting will require radical packaging designs and arrangement of the laser diode and phosphor target assemblies. To be able to manipulate the emission spectrum of devices based on LD induced lighting (LDIL) the type of phosphor targets and the level of impurities constituting the phosphor target material will need to be studied and adjusted. Recent research has demonstrated the necessity of these studies by investigating the structural and thermal properties of $\mathrm{Tb}$, Ce doped $\mathrm{Y}_{3} \mathrm{Al}_{5} \mathrm{O}_{12}$ (YAG) single crystals. ${ }^{13,14}$ The results act as pointers to the research that needs to be undertaken in parallel to both develop radical designs of LDIL modules and the associated single crystals used as emitting targets. In this report we have focused on studying different crystal sizes of the same phosphor material with the same LDs. We have carried out studies on beam incident angle and relationship between the emission properties and the beam power assembly solutions. We have previously reported on such solutions by making phosphor/ceramic composites with high thermal conductivity incorporated into various packaging solutions. ${ }^{15,16}$ Unlike LED's, the conversion process from LDs is in reflective mode rather than transmission through the phosphor target which can be challenging when packaging constraints are considered. Our target designs were based on YAG phosphor powder materials in a ceramic composite, ${ }^{15,16}$ however recently we have turned our attention to considering the application of single crystals of YAG:Ce (these have become readily available on the market)

\footnotetext{
*Electrochemical Society Member.
}

${ }^{\text {z} E-m a i l: ~ a l i . s a l i m i a n @ ~ b r u n e l . a c . u k ~}$ which possess high thermal conductivity. ${ }^{14,17}$ The advantage of single crystal applications is that unlike synthesized phosphor powder/ ceramic composite targets, the crystals can be polished to specific roughness values, hence enabling light harvesting from various planes of the target and simplifying the challenges associated to packaging of such lighting modules. The objective of this research was to exactly address the practicality of YAG single crystals in laser induced lighting applications. These experiments were conducted to obtain a thorough understanding of YAG single crystal's emission characteristics when activated by high laser beam powers of up to $>14 \mathrm{~W}$. Exposing these targets to high laser powers requires the material to possess efficient thermal conductivity along with appropriate thermal management.

\section{Experimental}

To obtain an insight into the emission characteristics of the single crystal YAG: Ce samples various tests were carried out. Five single crystals with similar dimensions and various thicknesses were tested. The samples were provided by CRYTUR, spol.s.r.o. All samples were $10 \mathrm{~mm} \times 10 \mathrm{~mm}$ with thicknesses of $1 \mathrm{~mm}$ to $5 \mathrm{~mm}$. The samples demonstrated homogenous Cerium distribution within the lattice. The exact amount of Cerium content in the samples is commercially protected and not disclosed by CRYTUR. The two front faces of the crystals were polished by the manufacturer while the sides were not polished to the same level of roughness. The surface roughness of the crystals was measured with a Zygo white light interferometer. The emission and excitation characteristics of each individual crystal were examined using a purpose built phosphor measurement system obtained from Bentham instruments UK. The laser beam/crystal emission studies were conducted by initially establishing a relationship between the beam power and the luminous flux of the crystals by applying high laser powers of up to $14.4 \mathrm{~W}$. The effect of incident angle of the laser beam on each YAG: Ce crystal was investigated by carrying out tests at 90 and 45 degrees. To obtain a comprehensive understanding of the emission characteristics of the single crystal's surface when excited by the laser; certain tests were carried out by masking various faces of the crystals when exposed to the laser beam. Finally by focusing and defocusing the beam to rectangular beam sizes of $\sim 1 \mathrm{~mm} \times 1 \mathrm{~mm}$ and $\sim 4 \mathrm{~mm} \times 1.5 \mathrm{~mm}$ the effect of focusing the beam energy on emission properties was investigated. Prior to the experiments, the samples were washed and cleaned using a $2 \%$ Helmanex solution in water. They were then washed and sonicated in an acetone bath for 10 minutes followed by washing and sonication in methanol and finally the surface was treated using an $\mathrm{O}_{2} / \mathrm{Ar}$ radio frequency plasma for 10 minutes. 


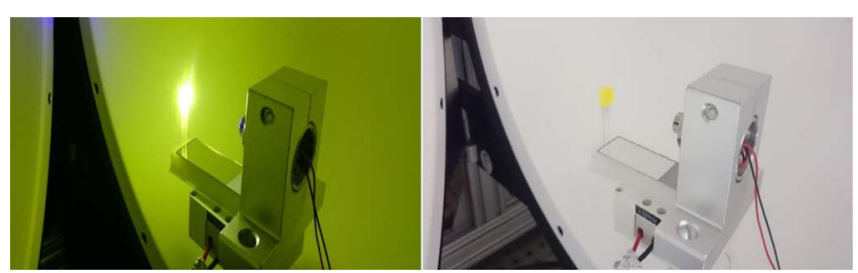

Figure 1. Set up of the sample holder 'A' with one laser diode. (Left: On, Right: Off)

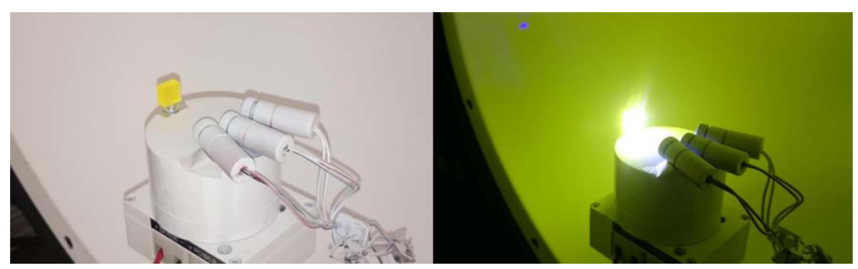

Figure 2. Set up of the sample holder 'B' with three laser diodes. (Right: On, Left: Off)

Surface analysis.-Surface analysis of the samples was simply conducted by placing the samples in the focal point of a zygo white light interferometer lens and the surface roughness was obtained.

Emission and excitation study.-The emission and excitation study of the samples was conducted by masking the sides and back of the crystal with sticky aluminum foil and placing them in the dark chamber of a Bentham spectrophotometer.

Laser beam induced emission.-For laser interaction studies, each sample was mounted as such to ensure minimum contact between the crystal and sample stand (only the bottom plane was covered by the sample stand). Two sample holders were designed (Figs. 1 and 2); one for testing the crystals with a single laser diode (A) and another with three diodes (B) to examine the emission properties of the diode when exposed to extremely high laser beam powers (above $14 \mathrm{~W}$ ). The sample holders where placed inside the central sample holder of an integrating sphere. Photometric analysis of the emission was carried out by placing the test lamps inside a 'Labsphere LMS200' model integrating sphere with diameter of 20 inches. This sphere is capable of analyzing test lamps with output of up to 8000 lumens. The sphere was calibrated using an AS-01331-000 test lamp provided by the manufacturer. The correction to account for light absorbed by the test lamp body was carried out using an auxiliary lamp inside the sphere which calculated the light absorbed by the test lamp body which was automatically factored into the measurements. We have previously reported on the type and structure of the laser diodes used in this study. ${ }^{15}$

\section{Results and Discussion}

Surface roughness analysis.-Figure 3 demonstrates the results obtained from the white light interferometer measurements. Surface roughness is a component of surface texture and is quantified by the deviations in the direction of the normal vector of the surface from its ideal form. The surface roughness of the crystals ranges from 770 to 310 nanometers. It is important to consider certain roughness for the surface of the crystals as an ultimately smooth surface may deflect some of the laser beam and undermine the efficiency.

Emission and excitation analysis of the crystals. - To ensure that the light escaping from the sides of the crystal did not interfere with the measurement, all sides except the front face of the crystals were masked with aluminum tape. The excitation spectra were obtained based on the $537 \mathrm{~nm}$ emission peak. All crystals manifested similar excitation spectra as illustrated in Figure 4 (which is the excitation spectrum of the $1 \mathrm{~mm}$ thick crystal). The emission spectra of all the crystals were similar; the emission spectrum of the $1 \mathrm{~mm}$ thick crystal excited under various wavelengths is presented in Figure 5. Clearly as expected from the excitation spectra presented in Figure 4 the optimum excitation wavelength for the YAG:Ce crystals is around $460 \mathrm{~nm}$

Laser power relationship.-The structure of the sample stand and the laser sources is shown in Figure 1 (setup up A) and Figure 2 (set up B). The laser power \& emission intensity tests were carried out using the sample holder 'B' setup. The $5 \mathrm{~mm}$ thick crystal was exposed to various combinations of beam powers using the three diodes. The beam diameter of all diodes was adjusted to $1 \mathrm{~mm} \times 1 \mathrm{~mm}$ rectangular spot. The diodes were adjusted so that each targeted a different spot on the surface of the crystal. The central diode was at 90 degrees while the other diodes targeted the sample at 70 degrees. Using on and off combination of the three diodes the sample was exposed to four different beam powers and the average luminous flux related to the total beam power targeting the phosphor was calculated, these results along with the standard deviations are illustrated in Table I and the luminous flux is plotted against the laser beam power in Figure 6 where there seems to be linear relationship between the beam power and luminous flux output. This test was carried out for with the 2, 3 and $4 \mathrm{~mm}$ crystals as well and a similar linear relationship was observed (not reported here).

Laser beam angle of incidence and beam size effect.-Using the sample holder 'A' setup (Figure 1), all the crystals were tested with two different laser beam focal adjustments of $(1 \mathrm{~mm} \times 1 \mathrm{~mm})$ and

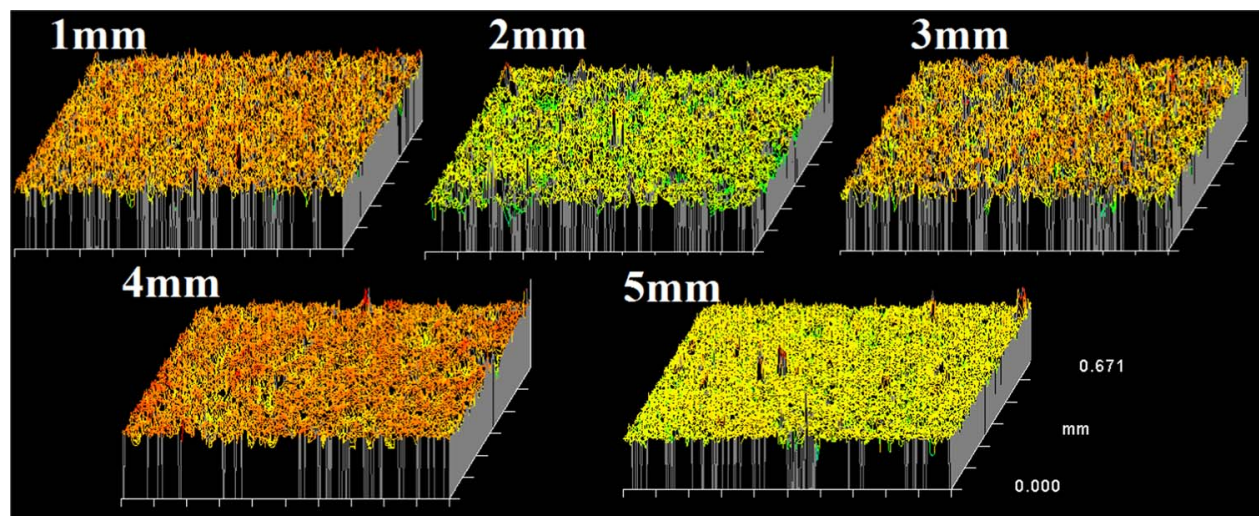

Figure 3. Surface analysis of the Crystals, the Ra value of the front face of the crystal exposed to the laser beam was measured. The crystals demonstrated an average Ra value of $546 \mathrm{~nm}$ with standard deviation value of 184 . 




Figure 4. Excitation spectrum of the $1 \mathrm{~mm}$ thick crystal. The ripples around the $468 \mathrm{~nm}$ main peak are artefacts associated to the spectrometer's xenon lamp.

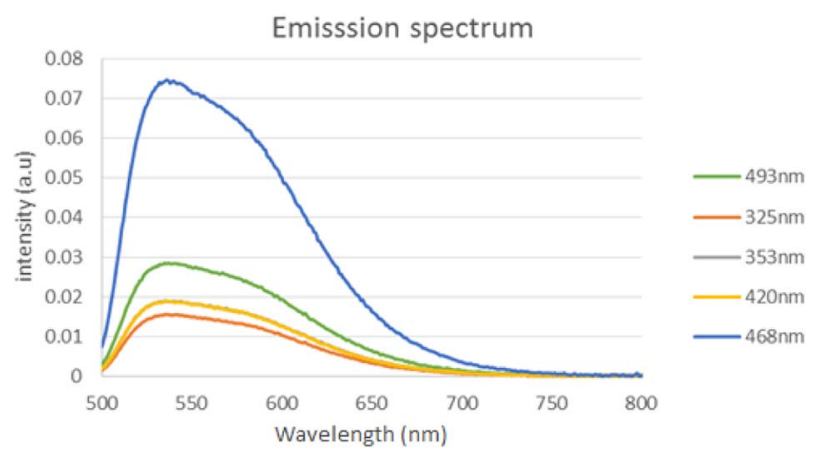

Figure 5. Emission spectra of the $1 \mathrm{~mm}$ thick crystal excited under the wavelengths shown in the figure. It can be seen that the phosphor target is most responsive to the $468 \mathrm{~nm}$ excitation wavelength as also illustrated in Figure 4.

Table I. Luminous flux output and associated exciting laser power.

\begin{tabular}{ccc}
$\begin{array}{c}\text { Laser } \\
\text { power }(\mathrm{mW})\end{array}$ & $\begin{array}{c}\text { Average Luminous } \\
\text { flux }(\mathrm{m})\end{array}$ & $\begin{array}{c}\text { Standard } \\
\text { deviation }\end{array}$ \\
\hline 14400 & 4256 & 9.4 \\
9600 & 2855 & 9.0 \\
6200 & 1840 & 8.9 \\
4900 & 1398 & 14.8
\end{tabular}

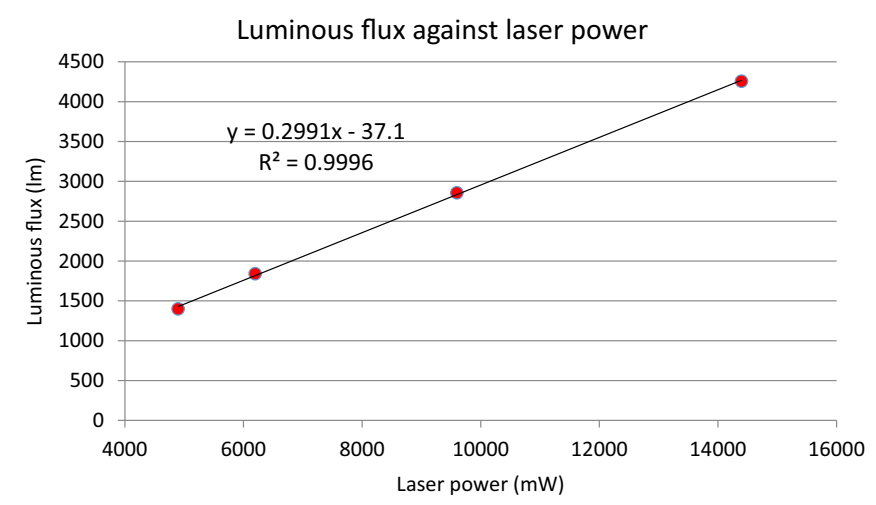

Figure 6. Luminous flux from the $5 \mathrm{~mm}$ thick crystal plotted against the exciting laser power.
Table II. One millimeter beam size (1 $\mathrm{mm} \times 1 \mathrm{~mm})$, Beam power $\sim 6.5 \mathrm{~W}$.

\begin{tabular}{|c|c|c|c|c|}
\hline \multirow[b]{2}{*}{ Crystal thickness } & \multicolumn{4}{|c|}{$\begin{array}{c}\text { Luminous flux }(\mathrm{lm}) \\
\text { Laser Beam incident angle }\end{array}$} \\
\hline & \multicolumn{2}{|c|}{45 Degrees } & \multicolumn{2}{|c|}{90 Degrees } \\
\hline $1 \mathrm{~mm}$ & \multicolumn{2}{|c|}{1847} & \multicolumn{2}{|c|}{1885} \\
\hline $2 \mathrm{~mm}$ & \multicolumn{2}{|c|}{1908} & \multicolumn{2}{|c|}{1942} \\
\hline $3 \mathrm{~mm}$ & \multicolumn{2}{|c|}{1847} & \multicolumn{2}{|c|}{1922} \\
\hline $4 \mathrm{~mm}$ & \multicolumn{2}{|c|}{1857} & \multicolumn{2}{|c|}{1919} \\
\hline \multirow[t]{2}{*}{$5 \mathrm{~mm}$} & & & & \\
\hline & \multicolumn{4}{|c|}{ Chromaticity } \\
\hline Crystal thickness & \multicolumn{2}{|c|}{45 Degrees } & \multicolumn{2}{|c|}{90 Degrees } \\
\hline $1 \mathrm{~mm}$ & 0.373 & 0.446 & 0.392 & 0.479 \\
\hline $2 \mathrm{~mm}$ & 0.368 & 0.429 & 0.387 & 0.467 \\
\hline $3 \mathrm{~mm}$ & 0.363 & 0.417 & 0.390 & 0.468 \\
\hline $4 \mathrm{~mm}$ & 0.367 & 0.421 & 0.392 & 0.470 \\
\hline $5 \mathrm{~mm}$ & 0.367 & 0.419 & 0.400 & 0.485 \\
\hline
\end{tabular}

$(4 \mathrm{~mm} \times 1.5 \mathrm{~mm})$. The tests were carried out with the beam striking the crystals at 90 and 45 degrees. The luminous flux and the chromaticity of the emission from the crystals were recorded. The data are presented in Tables II and III.

From Tables II and III, it can be seen that the 90 degrees incident angle generates better values for luminous flux than the 45 degrees. This is simply due to the reflection of the laser and hence wasting of the laser energy at the 45 degrees incident angle. The values for the chromaticity in both tables clearly demonstrate some of the blue laser light escaping off the surface of the phosphor crystal influencing the color temperature of the resulting "white light". It is clear, however that the excitation angle influences the CIE coordinates and for desired color temperatures the choice of this angle may be critical. It also indicates that when designing a LD based solid lighting solution, one needs to consider all aspects of the laser reflections off the phosphor target to ensure safety of the design by preventing any of the laser beam escaping. Comparing the results presented in Table III where a larger beam was evaluated, there is little evidence for much difference in brightness. In terms of phosphor performance, focusing or defocusing the beam does not seem to make a significant difference in brightness, however the incident angle is critical in both cases.

The plot of the chromaticity coordinates (Tables II and III) which are presented in Figure 7 clearly demonstrates that more of the blue light is present in the total emission at the 45 degrees incidence angle. The $1 \mathrm{~mm}$ thick crystals seems to favor emission toward warmer color 
Table III. Four millimeter beam size $(4 \mathrm{~mm} \times 1.5 \mathrm{~mm})$, Beam power $\sim 6.5 \mathrm{~W}$.

Luminous flux (lm)

Laser Beam incident angle

\begin{tabular}{|c|c|c|c|c|}
\hline \multirow[b]{2}{*}{ Crystal thickness } & & & & \\
\hline & \multicolumn{2}{|c|}{45 Degrees } & \multicolumn{2}{|c|}{90 Degrees } \\
\hline $1 \mathrm{~mm}$ & \multicolumn{2}{|c|}{1782} & \multicolumn{2}{|c|}{1861} \\
\hline $2 \mathrm{~mm}$ & \multicolumn{2}{|c|}{1862} & \multicolumn{2}{|c|}{1891} \\
\hline $3 \mathrm{~mm}$ & \multicolumn{2}{|c|}{1869} & \multicolumn{2}{|c|}{1908} \\
\hline $4 \mathrm{~mm}$ & \multicolumn{2}{|c|}{1860} & \multicolumn{2}{|c|}{1909} \\
\hline $5 \mathrm{~mm}$ & \multicolumn{2}{|c|}{1847} & \multicolumn{2}{|c|}{1878} \\
\hline & \multicolumn{4}{|c|}{ Chromaticity } \\
\hline Crystal thickness & \multicolumn{2}{|c|}{45 Degrees } & \multicolumn{2}{|c|}{90 Degrees } \\
\hline $1 \mathrm{~mm}$ & 0.382 & 0.459 & 0.390 & 0.480 \\
\hline $2 \mathrm{~mm}$ & 0.366 & 0.424 & 0.382 & 0.455 \\
\hline $3 \mathrm{~mm}$ & 0.371 & 0.433 & 0.385 & 0.462 \\
\hline $4 \mathrm{~mm}$ & 0.367 & 0.423 & 0.392 & 0.474 \\
\hline $5 \mathrm{~mm}$ & 0.378 & 0.439 & 0.392 & 0.468 \\
\hline
\end{tabular}

temperatures. This may suggest that the thickness of the crystal needs to be selected and optimized for the color temperature and brightness desired. It should be stressed that the variation in the chromaticity coordinates in both the 45 degree incidence angle group and in the 90 degree incidence group are small overall. Total Crystal surface area and emission

The $(1 \times 1 \mathrm{~mm})$ beam size and $x \& y$ chromaticity coordinates at 90 and 45 degrees laser incident angles

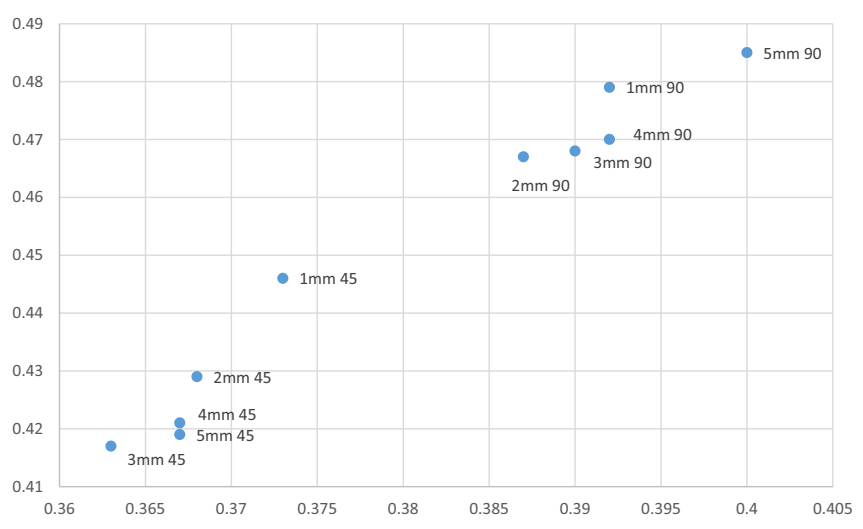

The $(4 X 1.5 \mathrm{~mm})$ beam size and $x \& y$ chromaticity coordinates at 90 and 45 degrees laser incident angles



Figure 7. Chromaticity coordinates at different incident angles and beams size for $1 \mathrm{~mm}$ to $5 \mathrm{~mm}$ thick crystals. (Top: $1 \mathrm{~mm} \times 1 \mathrm{~mm}$ beam, Bottom: $4 \mathrm{~mm} \times 1.5 \mathrm{~mm}$ beam)

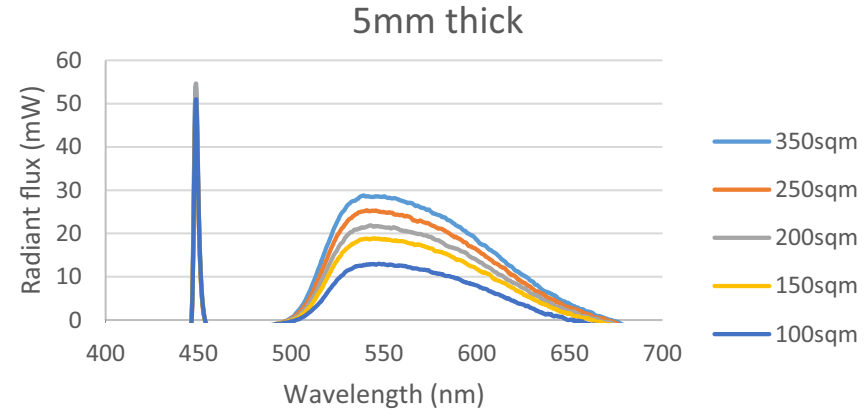

Figure 8. Radiant flux against total exposed surface area of the $5 \mathrm{~mm}$ thick crystal The masking of the crystal sides affects the emission intensity as light will get entrapped within the crystal.

To gain an understanding of emission characteristics from the surface of the crystal, a series of tests were carried out using a $4.3 \mathrm{~W}$ laser beam power in a type ' $B$ ' set up with a beam diameter of $1 \mathrm{~mm}$. To see the effect of emission from a given crystal the total emitting surface of the crystal could be controlled by masking one or more faces. Aluminium tape was used so that some of the light that hit a given surface was not absorbed and could be emitted from the open surfaces. This allowed the experiments to be carried out without making adjustments to the total calibrations of the integrating sphere. The tests were carried out so that at each stage one or more of the crystal faces was masked. Given the dimensions of the crystals, the tests were carried out to enable the plotting of the luminous flux of the crystal's emission against the total uncovered surface of the crystal. For example, using the $5 \mathrm{~mm}$ thick crystal, the total area exposed without any masking was calculated as follows:

Total area $=\sum$ (f1) (f2) (s1) (s2) (s3)

Where (face 1 and face 2 ) $\mathrm{f} 1 \& \mathrm{f} 2=10 \times 10 \mathrm{~mm}=100 \mathrm{~mm}^{2}$ each.

(Side 1, 2 and 3) s1, s2 \& s3 $=10 \mathrm{~mm} \times 5 \mathrm{~mm}=50 \mathrm{~mm}^{2}$ each.

Giving a total exposed surface area of: $100+100+50+50+$ $50=350 \mathrm{~mm}^{2}$ for the $5 \mathrm{~mm}$ thick crystal.

The bottom side where the sample was rested on a flat holder was ignored in calculation for all samples. The plot of Radiant flux value against total unmasked surface area is presented for the $5 \mathrm{~mm}$ thick crystal in Figure 8.

The observed relationship between the Luminous flux ( $\mathrm{lm})$ and the total surface area of the crystal is presented in Figure 9 for the 2,3,4 and $5 \mathrm{~mm}$ thick crystals.

The results presented in Figure 9 clearly demonstrate the role importance of light trapping within the crystal body.

High triple beam power tests._Using the ' $\mathrm{B}$ ' sample holder configuration (Figure 2) where one crystal was exposed to three laser diode beams the overall output of each crystal was evaluated. The total beam power incident on the crystals was $\sim 14 \mathrm{~W}$. The results are presented in Table IV.

Although each crystal seems to have slightly different chromaticity coordinates, there is no particular trend that indicates a direct relationship between the crystal size and the chromaticity, however it must be stressed that we did not attempt to saturate the crystals and we exposed them to the same beam power. This probably means the number of activator sites in the crystals play a role in the conversion of some light into heat by various quenching mechanisms. Above we demonstrated the importance of the incident angle on the chromaticity. Considering that the results presented in Figure 10 were obtained by a combination of three laser beams incident at the crystal there could be slight deviations in precise measurement of the angles. To carry out this objective more accuratly, a new sample holder and laser modules should be designed allowing a high degree of control over angle of incidence and precise positioning of the single crystal which was beyond the 



Figure 9. The relationship between the Luminous flux (lumens) and the total surface area of the 2,3,4 and $5 \mathrm{~mm}$ thick crystal.

Table IV. Emission characteristics of the crystals with maximum available laser power of $\sim 14 \mathrm{~W}$ using the sample holder 'B' set up.

\begin{tabular}{|c|c|c|c|c|c|c|c|}
\hline \multirow[b]{2}{*}{ Crystal thickness } & \multirow[b]{2}{*}{ Radiant Flux (mW) } & \multirow[b]{2}{*}{ Luminous Flux (lm) } & \multirow[b]{2}{*}{ SD } & \multirow[b]{2}{*}{ Color Temperature $(\mathrm{K})$} & \multirow[b]{2}{*}{ Color Rend. Index } & \multicolumn{2}{|c|}{ Chromaticity } \\
\hline & & & & & & $\mathbf{x}$ & $\mathbf{y}$ \\
\hline $5 \mathrm{~mm}$ & 9417.00 & 4230 & 10.1 & 4166 & 53.2 & 0.4011 & 0.4888 \\
\hline $4 \mathrm{~mm}$ & 9260.00 & 4202 & 12 & 4121 & 53.3 & 0.4061 & 0.4986 \\
\hline $3 \mathrm{~mm}$ & 9752.00 & 4242 & 9.5 & 4337 & 54.3 & 0.3871 & 0.4671 \\
\hline $2 \mathrm{~mm}$ & 9651.00 & 4291 & 11.3 & 4224 & 54.3 & 0.3974 & 0.4868 \\
\hline
\end{tabular}

objectives of this initial project on evaluating the potential of these single crystals.

\section{Conclusions}

The emission and excitation spectra of the crystals demonstrates that they behave similarly to phosphor powders of YAG:Ce. The thick-

The $x$ \& y Chromaticity coordinates of the crystals tested using lamp B

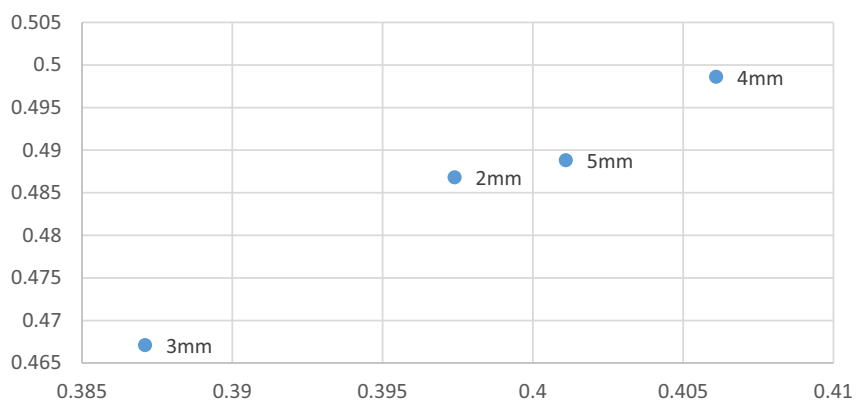

Figure 10. Chromaticity coordinates of 2,3,4 and $5 \mathrm{~mm}$ thick crystals excited with $14 \mathrm{~W}$ of laser beam under lamp B configuration. ness of the crystals does not affect their excitation or emission properties, though they do affect the CIE coordinates without a particular trend. However, it's interesting to notice that the size of the crystals does not seem to be affecting the total luminous flux of the samples as one may expect larger crystals to have brighter output. Specifically when the results presented in Figure 9 are considered where one can clearly observe the amount of light that is escaping from the sides of the crystals when excited by the laser beam. This is possibly due to trapping some of the generated emission within the thicker crystals. So, although theoretically a larger crystal may emit more light at higher emission intensities, at the same time its thickness and hence the path the light takes to leave the crystal bulk is also increased and so one factor may balance the other factor. This can be further investigated by polishing all sides of the crystals to roughness values in the region of a few nanometers compared to their current state. The incident beam angle is an important issue to consider. When the beam targets the surface of the crystal at an angle lower than 90 degrees, there seems to be some waste of beam power due to reflection off the crystal surface, clearly the light needs to enter the crystal to excite it, and so if part of it does not then power is wasted. This is particularly important when considering a laser diode/phosphor target packaging solution of a potential light source. The ideal option would be to target the crystal with the light at 90 degrees to the surface, however such an approach may face certain restrictions in designing the light engines. Possible application of antireflective coatings or optimal surface roughness are topics that warrant further investigation to minimize the emission loss 
due to targeting the phosphor at angles lower than 90 degrees. We have previously reported on the importance of thermal conductivity of any phosphor target that may find application in the field of laser induced lighting systems especially when high laser powers are considered. As discussed we exposed these samples to laser powers of up to $14 \mathrm{~W}$. Provided that the crystals are thermally managed through application of a heat sink, their emission stability seems unaffected by the thermal energy of the beam that is imposed on them (this will be further investigated \& reported in the near future).

We have shown herein that single crystal YAG based phosphors offer an opportunity for the future of high brightness lighting solutions. Further work will need to be conducted to investigate how the composition of the single crystal phosphors, surface features, shape and morphology can affect their emission properties when excited by focused high power laser beams.

\section{Acknowledgment}

We thank Brunel University London for allocation of funds from EPSRC grant No. EP/K504208/1 \& APEX phase II program.

\section{References}

1. O. B. Shchekin, P. J. Schmidt, F. Jin1, N. Lawrence, K. J. Vampola, H. Bechtel, D. R. Chamberlin, and R. Mueller-Mach and G. O. Mueller, "Excitation dependent quenching of luminescence in LED phosphors," Phys. Status Solidi RRL., 201600006, (2016)

2. M.-H. Kim, M. F. Schubert, Q. Dai, J. K. Kim, E. F. Schubert, J. Piprek, and Y. Park, "Origin of efficiency droop in GaN-based light-emitting diodes," Appl. Phys. Lett., 91(18), 183507 (2007).

3. E. Kioupakis, P. Rinke, K. T. Delaney, and C. G. Van de Walle, "Indirect Auger recombination as a cause of efficiency droop in nitride light-emitting diodes," Appl. Phys. Lett., 98(16), 161107 (2011).
4. H.-Y. Ryu, D.-S. Shin, and J.-I. Shim, "Analysis of efficiency droop in nitride lightemitting diodes by the reduced effective volume of InGaN active material," Appl. Phys. Lett., 100(13), 131109 (2012).

5. J. Iveland, L. Martinelli, J. Peretti, J. S. Speck, and C. Weisbuch, "Direct measuremen of auger electrons emitted from a semiconductor light-emitting diode under electrical injection: Identification of the dominant mechanism for efficiency droop," Phys. Rev. Lett., 110(17), 177406 (2013).

6. A. David and M. J. Grundmann, "Droop in InGaN light-emitting diodes: A differential carrier lifetime analysis," Appl. Phys. Lett., 96(10), 103504 (2010).

7. J. Piprek, "Efficiency droop in nitride-based light-emitting diodes," Phys. Statu Solidi A, 207(10), 2217 (2010).

8. J. Hader, J. V. Moloney, and S. W. Koch, "Density-activated defect recombination as a possible explanation for the efficiency droop in GaN-based diodes," Appl. Phys. Lett., 96(22), 221106 (2010)

9. S. Grzanka, P. Perlin, R. Czernecki, L. Marona, M. Boćkowski, B. Łucznik M. Leszczyński, and T. Suski, "Effect of efficiency "droop" in violet and blue InGaN laser diodes," Appl. Phys. Lett., 95(7), 071108 (2009).

10. Y. Narukawa, M. Ichikawa, D. Sanga, M. Sano, and T. Mukai, "White light emitting diodes with super-high luminous efficacy," J. Phys. D Appl. Phys., 43(35), 354002 (2010).

11. J. J. Wierer Jr., J. Y. Tsao, and D. S. Sizov, "Comparison between blue lasers and light-emitting diodes for future solid-state lighting," Laser Photonics Rev., 7(6), 963 (2013).

12. J. J. Wierer Jr., J. Y. Tsao, and D. S. Sizov, "The potential of III-nitride laser diodes for solid-state lighting," Phys. Status Solidi, C Conf. Crit. Rev., 11(3-4), 674 (2014).

13. M. Faheem and K. Lynn, "Structural and thermal properties of Tb,Ce Doped $\mathrm{Y}_{2.97} \mathrm{Gd}_{0.03} \mathrm{Al}_{2} \mathrm{Ga}_{3} \mathrm{O}_{12}$ Single Crystals," American Journal of Analytical Chemistry, 5, 695 (2014).

14. S. Kostić, Z. Ž. Lazarević, V. Radojević, A. Milutinović, M. Romčević, N. Ž. Romčević, and A. Valčić, "Study of structural and optical properties of YAG and Nd:YAG single crystals," Materials Research Bulletin, 63, 80 (2015).

15. A. Salimian, J. Silver, G. R. Fern, M. Evans, and R. Haghpanahan, "Evaluation of thermally stable phosphor screens for application in Laser diode excited high brightness white light modules," ECS J. Solid State Sci. Technol., 5(1), R3001 (2016).

16. A. Salimian, G. R. Fern, H. Upadhyaya, and J. Silver, "Laser Diode Induced Lighting Modules," ECS J. Solid State Sci. Technol., 5(3), R26 (2016).

17. M. Cantore, N. Pfaff, R. M. Farrell, J. S. Speck, S. Nakamura, and S. P. DenBaars, "High luminous flux from single crystal phosphor-converted laser-based white lighting system," Optics Express, 24(2), 215 (2016). 